\title{
Nonsense-mediated mRNA decay modulates clinical outcome of genetic disease
}

\author{
Mehrdad Khajavi ${ }^{1}$, Ken Inoue ${ }^{2}$ and James R Lupski ${ }^{*, 1,3,4}$
}

${ }^{1}$ Department of Molecular and Human Genetics, Baylor College of Medicine, Houston, TX, USA; ${ }^{2}$ Department of Mental Retardation and Birth Defect Research, National Institute of Neuroscience, National Center of Neurology and Psychiatry, Tokyo, Japan; ${ }^{3}$ Department of Pediatrics, Baylor College of Medicine, Houston, TX, USA;

${ }^{4}$ Texas Children's Hospital, Houston, TX, USA

The nonsense-mediated decay (NMD) pathway is an mRNA surveillance system that typically degrades transcripts containing premature termination codons (PTCs) in order to prevent translation of unnecessary or aberrant transcripts. Failure to eliminate these mRNAs with PTCs may result in the synthesis of abnormal proteins that can be toxic to cells through dominant-negative or gain-of-function effects. Recent studies have expanded our understanding of the mechanism by which nonsense transcripts are recognized and targeted for decay. Here, we review the physiological role of this surveillance pathway, its implications for human diseases, and why knowledge of NMD is important to an understanding of genotype-phenotype correlations in various genetic disorders.

European Journal of Human Genetics (2006) 14, 1074-1081. doi:10.1038/sj.ejhg.5201649; published online 7 June 2006

Keywords: premature termination codons; nonsense-mediated decay; genotype-phenotype correlations

\section{Introduction}

The elucidation of nonsense-mediated decay (NMD) as an RNA surveillance system is a relatively recent event. Studies in yeast and other model organisms have enumerated specific molecular details that provide some insight into mechanisms whereby a transcript that contains a premature termination codon (PTC) is recognized by NMD factors and rapidly degraded, thus eliminating abnormal transcripts. ${ }^{1}$ Genetic screens in Saccharomyces cerevisiae and Caenorhabditis elegans have identified key NMD factors such as UPF1-3 (up-frameshift proteins 1, 2 and 3) in yeast and smg1-7 (suppressor with morphogenetic effects on genitalia 1-7) in C. elegans that play an essential role in NMD. ${ }^{2-5}$ The importance of UPF genes for higher life forms is highlighted by the observation that the basic function of

${ }^{*}$ Correspondence: Dr JR Lupski, Department of Molecular and Human Genetics, Baylor College of Medicine, One Baylor Plaza, Rm604B, Houston, TX 77030, USA.

Tel: + 1713798 6531; Fax: + 1713798 5073;

E-mail: jlupski@bcm.tmc.edu

Received 12 September 2005; revised 10 April 2006; accepted 11 April 2006; published online 7 June 2006 these proteins is conserved in all eukaryotes. The UPF1-3 proteins (also known as SMG2-4 in C. elegans) are conserved from yeast to humans, whereas SMG1, SMG5 and SMG6 have orthologs in higher eucaryotes but not in S. cerevisiae. ${ }^{6}$ Deletion or suppression of Upf genes eliminates NMD and prevents the normally rapid decay of transcripts containing nonsense or frameshift mutations in eucaryotes. ${ }^{3,7,8}$

The exact molecular mechanism for NMD in higher eucaryotes is still being elucidated. In mammalian NMD, an intron apparently functions as a second signal for triggering NMD by leaving a 'mark' on the mRNA at the exon-exon junction as a consequence of the splicing event. This mark known as the exon-junction complex (EJC) $)^{9,10}$ enables the NMD RNA surveillance pathway to differentiate between PTCs and normal stop codons present in the last exon ensuring the degradation only of transcripts containing nonsense codons that are followed by an intron. ${ }^{11,12}$ Evidence from a number of studies suggests that during the initial rounds of translation, referred to as the 'pioneer round', ${ }^{13}$ the translating ribosome pauses at the PTC. Release factors eRF1 and 
eRF3, which associate with the terminating ribosome, recruit UPF1, which subsequently makes contact with UPF2 and UPF3, which are bound to EJCs. ${ }^{9,14}$ Formation of this complete 'surveillance complex', containing UPF1-3 is believed to initiate mRNA degradation by NMD (for a review see Lejeune and Maquat ${ }^{15}$ ). In normal transcripts, in which the termination codon occurs in the final exon, all EJCs are displaced by the translating ribosomes. Thus, the complete surveillance complex cannot form during translation termination because of the absence of UPF2 and UPF3, thus NMD is avoided. One of the main roles of mammalian NMD is to regulate the expression of many physiological transcripts. ${ }^{2,15,16}$ Given the number and diversity of transcripts regulated by NMD, the mammalian NMD machinery may have evolved into a more complex and efficient system in comparison to other lower model organisms. Recently, it has been reported that Upf1 is not only essential to NMD but also is required both for rapid degradation of histone mRNAs and reducing the half-life of normal mRNAs containing a Staufen binding site. $^{17,18}$ This has created a unique dependence of mammals on the NMD pathway and its key factors.

The mammalian NMD surveillance system cannot distinguish PTCs in the penultimate exon that are located less than $\sim 55$ base pairs (bp) from the final intron. The ' $255 \mathrm{bp}$ rule' for the 3 ' end of the penultimate exon is believed to reflect the location of the EJC on spliced mRNAs; a translocating ribosome would displace an EJC upstream of the last exon-exon junction before the ribosome could recognize a stop codon located less than $\sim 55$ nucleotide from the exon-exon junction. ${ }^{9}$ However, there are several genes whose mRNA degradation appears to show exceptions to this rule, including hexoseaminidase A $(H E X A),{ }^{19} \mathrm{MPZ}$, encoding myelin protein zero, ${ }^{20} H N F-1 \beta$ (hepatocyte nuclear factor- $1 \beta$ ), ${ }^{21}$ and the T-cell receptor $(T C R)^{22}$ genes of the cellular immune system. The failure of some genes to follow the ' 55 boundary rule' suggest that either alternative or additional signals may exist other than the EJC positioned at the $\sim 20-\sim 24$ nucleotide upstream of the exon-exon junction or potentially an entirely different protein complex at the exon-exon junction could be utilized for some genes to trigger NMD. ${ }^{22}$ As a consequence, the inability to differentiate nonsense codon mutations in the last and $3^{\prime}$ end of the penultimate exon from the normal termination codon can result in the stable translation of mRNAs that contain PTCs located within these 'protected' regions. Thus, mRNAs with nonsense codons present in such positions can escape the NMD pathway (Figure 1). Such an 'escape from NMD surveillance' may cause expression of large amounts of aberrant truncated proteins with potential dominantnegative or gain-of-function effects in cells. This latter mechanism may be particularly relevant to 'disease genes' with large last exons.

There are many well-studied examples of human phenotypes resulting from nonsense or frameshift mutations that are modulated by NMD (Tables 1-3). The phenotypes of genetic diseases are likely to be frequently affected by NMD as nonsense and frameshift mutations are present in approximately one-third of point mutations that cause human genetic disorders. ${ }^{23,24}$ Here, we review the roles for the NMD RNA surveillance pathway in altering the inheritance pattern for a disease trait and in modifying the ultimate phenotype for selected human diseases.

\section{NMD and human Mendelian disease}

The variation in clinical severity caused by different mutations in a single gene can often be defined by the different effects of mutated protein. Generally, in vitro studies that assay and compare wild-type and mutant proteins have often enabled verification of the functional consequences causing pathogenic effects associated with each mutation in a single gene and sometimes define a

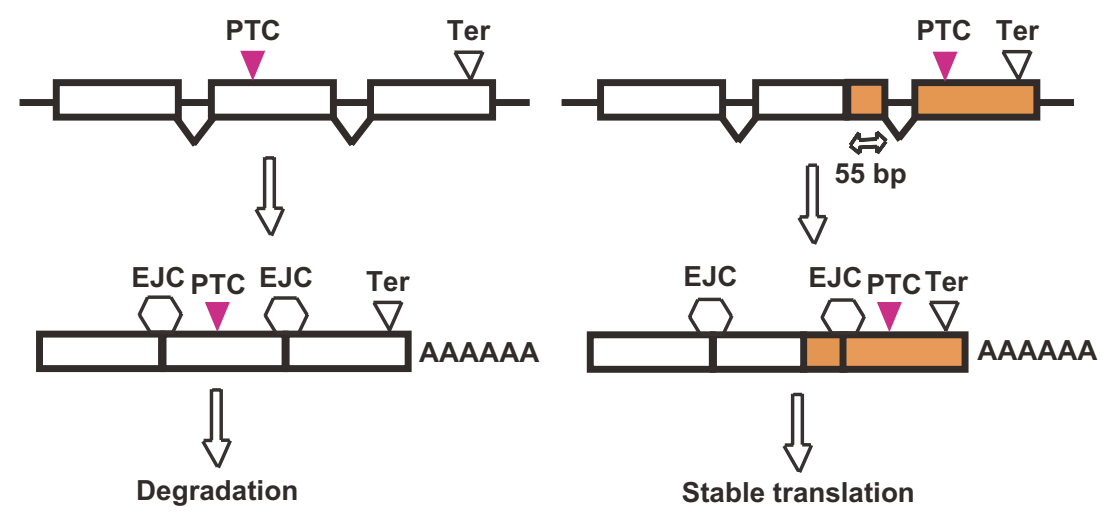

Figure 1 Simplified model of mammalian NMD. For transcripts containing a PTC, if the ribosome encounters an EJC downstream, NMD is triggered and leads to mRNA decay or degradation. In contrast, transcripts with PTCs in the $3^{\prime}$ portion of the gene (orange), including the last exon and $\sim 55 \mathrm{bp}$ of the penultimate exon, are stably translated into truncated proteins that could potentially result in a severe phenotype. 
Table 1 NMD altering the pattern of inheritance

\begin{tabular}{|c|c|c|c|c|}
\hline Gene name & Gene symbol & OMIM reference no. & $\begin{array}{l}\text { Phenotype: } 5^{\prime} \text { PTC } A R \\
\text { 3' PTC } A D\end{array}$ & References \\
\hline Hemoglobin- $\beta$ & $H B B$ & 141900 & $\beta$-Thalassemia & 25,26 \\
\hline Chloride channel 1, skeletal muscle & CLCN1 & 118425 & $\begin{array}{l}5^{\prime} \text { PTC Becker disease } \\
\text { 3' PTC Thomsen disease }\end{array}$ & 27 \\
\hline Rhodopsin & $\mathrm{RHO}$ & 180380 & Retinitis pigmentosa & 28,49 \\
\hline Cone-rod homeobox-containing gene & $C R X$ & 602225 & $\begin{array}{l}\text { Leber congenital amaurosis } \\
\text { 5' PTC heterozygous normal } \\
\text { 3' PTC AD }\end{array}$ & 29 \\
\hline Receptor tyrosine kinase-like orphan receptor 2 & $R O R 2$ & 602337 & $\begin{array}{l}5^{\prime} \text { PTC RRS } \\
3^{\prime} \text { PTC BDB1 }\end{array}$ & 30 \\
\hline ATP-binding cassette, subfamily C member 6 & $A B C C 6$ & 603234 & PXE & 34 \\
\hline
\end{tabular}

AR: autosomal recessive; AD: autosomal dominant; PTC: premature termination codon, genetic disorders; BDB1: brachydactyly type B1, PXE: pseudoxanthoma elasticum, RRS: robinow syndrome.

Table 2 NMD causing distinct traits to manifest from mutations in the same gene

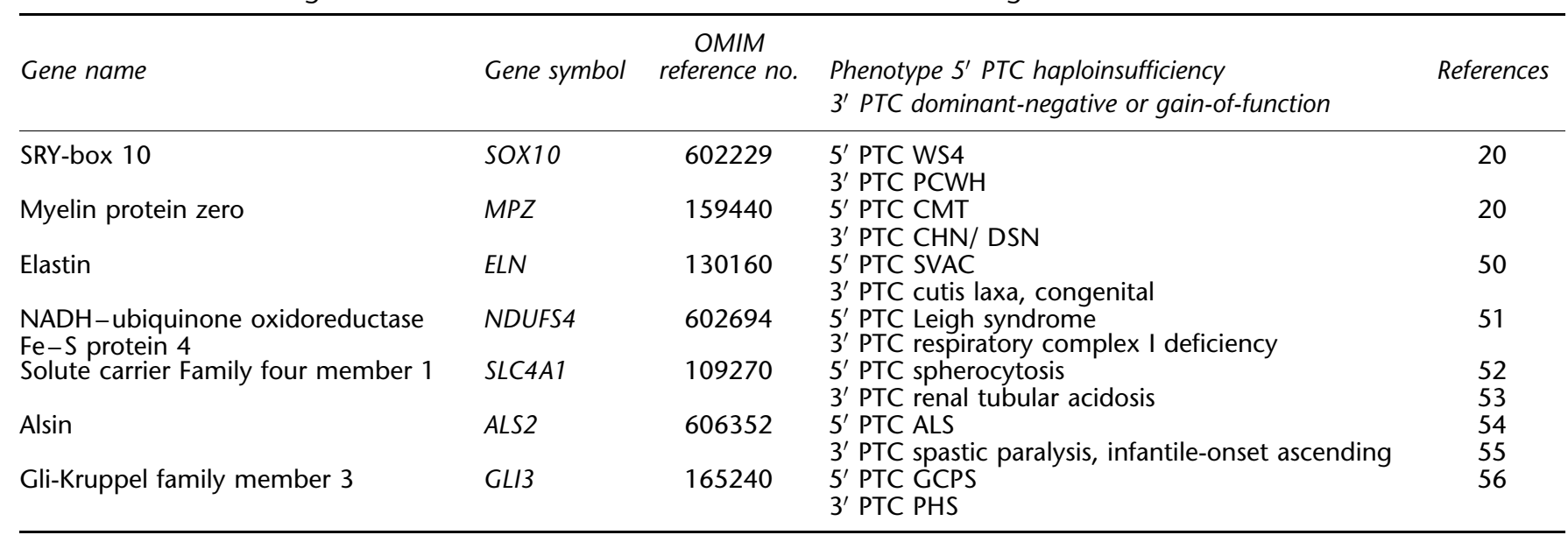

PTC: premature termination codon, genetic disorders; ALS: amyotrophic lateral sclerosis; CHN: congenital hypomyelinating neuropathy; CMT: Charcot-Marie-Tooth disease; DSN; Dejerine-Sottas neuropathy; GCPS: Greig cephalopolysyndactyly syndrome; PCWH: peripheral demyelinating neuropathy, central dysmyelinating leukodystrophy, Waardenburg syndrome and Hirschsprung disease; PHS: Pallister-Hall Syndrome; SVAC: supravalvular aortic stenosis, WS4: Waardenburg-Hirschsprung disease.

Table 3 NMD modifying the specific clinical phenotype

\begin{tabular}{|c|c|c|c|c|}
\hline Gene name & Gene symbol & OMIM reference no & Phenotype & References \\
\hline Collagen type I, $\alpha 1$ & COL1A1 & 120150 & $\begin{array}{l}\text { 5' PTC OI type I (mild) } \\
\text { 3' PTC OI type II-IV }\end{array}$ & $\begin{array}{l}40 \\
41\end{array}$ \\
\hline Collagen type II, $\alpha 1$ & COL2A1 & 120140 & $\begin{array}{l}\text { 5' PTC Stickler syndrome } \\
\text { 3' PTC spondylyeopiphyeal dysplasia }\end{array}$ & 42 \\
\hline Hexoseaminidase $\mathrm{A}$ & HEXA & 606869 & $\begin{array}{l}\text { Tay-Sachs disease } \\
5^{\prime} \text { PTC infantile (severe) }\end{array}$ & 43 \\
\hline Dystrophin & $D M D$ & 300377 & $\begin{array}{l}\text { Muscular dystrophy } \\
\text { 5' PTC severe } \\
\text { 3' PTC mild }\end{array}$ & 44 \\
\hline Paired box gene 6 & PAX6 & 607108 & $\begin{array}{l}\text { 5' PTC aniridia } \\
\text { 3' PTC no mutation detected }\end{array}$ & 57 \\
\hline Retinoblastoma & $R B 1$ & 180200 & $\begin{array}{l}\text { Retinoblastoma } \\
5^{\prime} \text { PTC early onset }\end{array}$ & 58 \\
\hline Ataxia-telangiectasia mutated gene & ATM & 607585 & $\begin{array}{l}\text { 5' PTC mild } \\
\text { 3' PTC severe- shorter survival }\end{array}$ & 59 \\
\hline
\end{tabular}

PTC: premature termination codon; OI: osteogenesis imperfecta. 
genotype-phenotype correlation. However, for some truncating mutations in vitro protein studies do not correlate with observed in vivo phenotypes (eg severe mutant protein effects were observed in vitro, but a relatively mild clinical phenotype was observed). Such mutant proteins were thought to be acting by dominant-negative or gainof-function effects based on functional assays, yet they conveyed a mild phenotype. Whereas some truncated proteins may have a deleterious effect in vitro, only mutant mRNA that escape NMD and result in mutant protein that is translated from a stable mRNA realize that potential in vivo. NMD usually prevents translation of transcripts containing $5^{\prime}$ premature stop codon that are followed by at least one intron, thereby reducing the amount of dominant-negative protein that could be produced and instead resulting in loss-of-function effects. NMD can effect clinical outcome in at least three major ways: (i) altering the pattern of inheritance, (ii) causing distinct traits to manifest from mutations in the same gene, and (iii) modifying the specific clinical phenotype.

\section{Dominant versus recessive traits conveyed by allelic truncating mutations}

The occurrence of both dominant and recessive mutant alleles in a single gene is unusual but not impossible. The process of NMD can be responsible for allelic mutations conveying phenotypes that segregate either as dominant versus recessive traits (Table 1 ). $\beta$-Thalassemia represents a classic example in which $5^{\prime}$ PTCs in the $\beta$-globin gene result in a recessive trait, whereas $3^{\prime}$ PTCs can result in an atypical dominant form of disease, because $5^{\prime}$ PTCs but not $3^{\prime}$ PTCs trigger $\beta$-globin NMD. ${ }^{25,26}$ In fact, although model organisms such as yeast and worms have helped elucidate the NMD pathway, the first clear example of NMD was with a human disease: $\beta$-thalassemia.

Similar disease and inheritance pattern modulating effects of NMD can explain genotype-phenotype correlations in a number of other human disorders such as myotonia congenita, ${ }^{27}$ retinal degeneration, ${ }^{28,29}$ Robinow syndrome $^{30}$ and brachydactyly-type $\mathrm{B}^{31}$ (Table 1 ). Mutations in the muscle chloride channel CLCN1 gene, which regulates the electrical excitability of the skeletal muscle membrane, can cause either dominant or recessive myotonia congenita. The autosomal recessive myotonia congenita (Becker disease) can be caused by compound heterozygous mutations, such as premature stop codons in an upstream exon of one allele in combination with a mutation in the other allele, causing a total loss-offunction in CLCN1. However, a less common Thomsen disease, the autosomal-dominant congenital myotonia, can be caused by PTCs in the last exon in one allele as such mutations may escape NMD and function as dominant-negative alleles. ${ }^{27}$
Interestingly, nonsense and frameshift mutations in ROR2, encoding a receptor tyrosine kinase, result in both an autosomal-recessive form of Robinow syndrome (RRS) and a dominant form of Brachydactyly type B (BDB). ${ }^{30,31}$ RRS is a severe skeletal dysplasia with shortening of the limbs, segmental defects of the spine, brachydactyly and a dysmorphic facial appearance, whereas BDB is characterized by distal phalanges and nail aplasia. ${ }^{30,32}$ No functional mechanisms have yet been delineated to explain effectively the association between mutation and different mode of inheritance causing different phenotypes (ie genotype/phenotype correlations). However, most mutations in ROR2 that results in premature stop codons in downstream exons appear to result in BDB segregating as an autosomal-dominant trait, whereas PTCs in upstream exons result in RRS. Previous studies ${ }^{33}$ have revealed decreased ROR2 mRNA levels in fibroblast cell lines of patients with PTCs causing the autosomal-recessive RRS, suggesting that these truncating mutations could possibly trigger the NMD pathway. By analogy to and inference from the $\beta$-globin gene observations, the decrease/absent mutant mRNA levels can cause a distinct recessive mode of inheritance.

Likewise, truncating mutations in $A B C C 6$, a member of ATP-binding cassette (ABC) transporter superfamily C, can potentially cause different patterns of inheritance (autosomal-recessive and a less common autosomaldominant form) for Pseudoxanthoma elasticum (PXE). NMD has been hypothesized to contribute to clinical variability in the expression of PXE. ${ }^{34}$ However, there has not been any direct evidence of its role in this disorder or as a mechanism to explain the phenotype or inheritance pattern from the genotype, or vice versa. This uncertainty has recently brought the existence of the autosomaldominant mode of inheritance of PXE into question. ${ }^{35}$

\section{Distinct phenotypic traits conveyed by allelic truncating mutations}

Although in theory all nonsense and frameshift mutations resulting in PTC are natural targets of the NMD RNA surveillance system, it is likely that NMD is not the only pathway actually responsible for mRNA stability/ degradation and thus modulating disease severity. Therefore, it is essential to verify any potential involvement of NMD experimentally, in order to investigate potential genotype-phenotype correlations and illuminate the molecular mechanisms underlying genetic disorders. Suppressing or ablating the expression of important NMD factors, such as hUpf1 and hUpf2, that can inhibit the NMD pathway directly ${ }^{8,20}$ is one experimental strategy to determine if NMD contributes to different mRNA expression levels and production of truncated proteins. Recent studies in two different peripheral neuropathy diseasecausing genes, SOX10 and MPZ, have shown that when 
either gene contains premature stop codons that escape $\mathrm{NMD}$, the translated proteins convey potent dominantnegative or gain-of-function effects. ${ }^{20,36-38}$ However, only transcripts with PTCs that escape NMD support that potential in vivo, as the activity of $5^{\prime}$ PTC is not realized because their expression is downregulated by the NMD RNA surveillance pathway. Thus, for each gene distinct neurological phenotypes may be conveyed owing to premature nonsense codons that trigger NMD versus those that escape NMD. ${ }^{20}$ The complex, severe, and extended neurological phenotype of peripheral demyelinating neuropathy, central dysmyelinating leukodystrophy, Waardenburg syndrome, and Hirschsprung disease (PCWH) is caused by SOX10 nonsense mutations that generate truncated mutant proteins possessing potent dominantnegative activity. However, the relatively milder phenotype that does not involve either the peripheral or the central nervous systems combines just Waardenburg and Hirschsprung diseases (WS4). This WS4 phenotype is caused by nonsense mutations that activate NMD, thereby reducing dominant-negative expression and resulting in haploinsufficiency (Figure 2). As in SOX10, different truncating mutations in $M P Z$ are responsible for distinct clinical entities that each affect the myelin of the peripheral nervous system. ${ }^{20}$ These neuropathies include early-onset congenital hypomyelinating neuropathy $(\mathrm{CHN})$ and Dejerine-Sottas neuropathy (DSN) as well as the less severe, adult onset Charcot-Marie-Tooth disease type 1B (CMT1B). It has been documented that the severity of some truncating alleles in CHN and DSN is owing to dominant-negative effects, whereas the reduced severity of truncating alleles in CMT1B is owing to loss-of-function. In essence, for PTC mutations in which the mRNA is degraded by NMD, this enables the conversion of dominant-negative or gain-of-function effects to haploinsufficiency (Table 2). Nevertheless, for selected genes such as MPZ, the PTC mutations that escape NMD may not always cause severe disease. Rather, the ultimate phenotype depends on the properties of the mutant proteins. ${ }^{39}$

\section{NMD can modify phenotypes conveyed by allelic truncating mutations}

The potential beneficial role of NMD is illustrated by genetic disorders whereby mutations usually convey a recessive trait, but can cause dominant diseases when the mutant transcripts escape NMD, and by disease genes in which toxicity of a mutant protein is mitigated by prevention of translation of the aberrant mRNA. However, NMD may also play a more subtle role in modulating
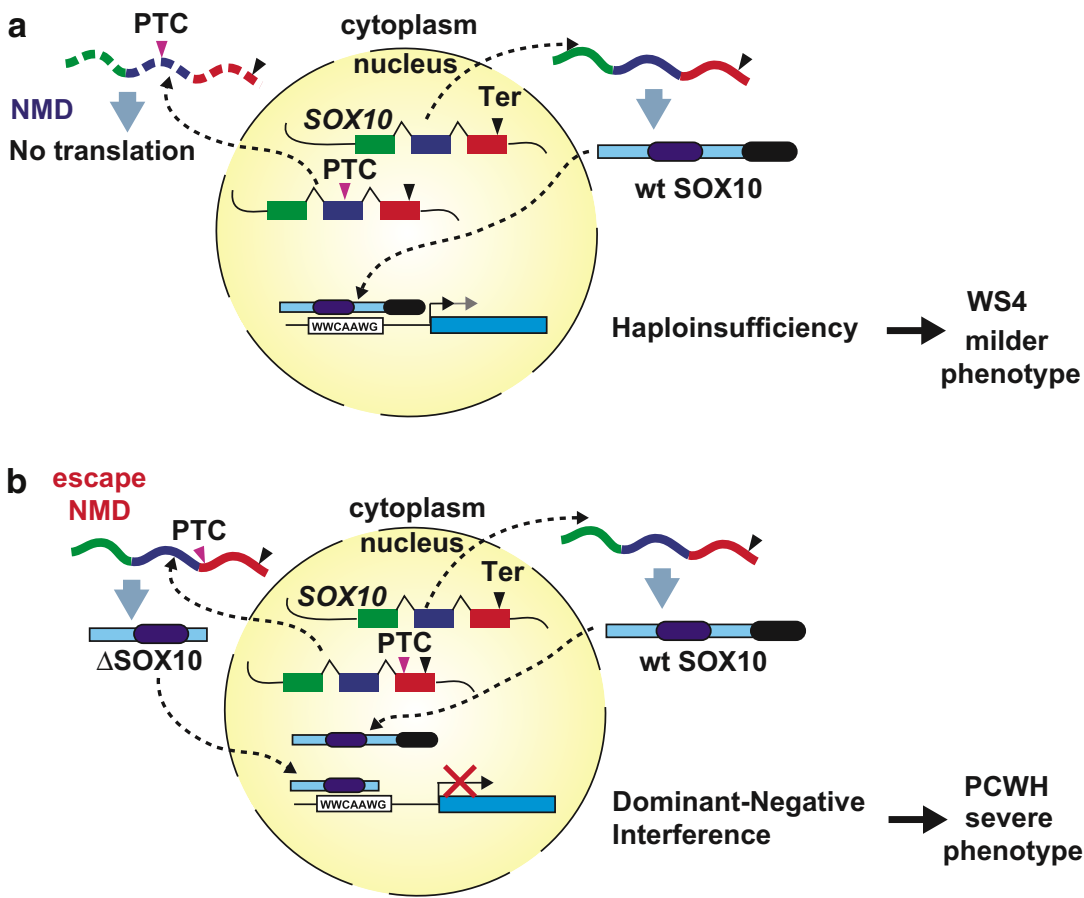

Figure 2 Schematic model of molecular mechanisms for PCWH and WS4. (a) WS4 is caused by SOX10 nonsense mutations that activate the protective NMD RNA surveillance pathway that results in the degradation of the mutant mRNAs containing $5^{\prime}$ PTCs before translation, thus mitigating the potential dominant-negative action of mutant proteins. As a result, these mutant alleles become null alleles and thus haploinsufficiency is the underlying mechanism causing the disease phenotype. (b) By contrast, mRNAs with nonsense mutations in the final exon escape NMD as there is no intron downstream of the premature stop codon. Large amounts of mutant truncated protein are produced. Such mutant protein can have dominantnegative or gain-of-function effects resulting in a severe PCWH disease phenotype. 
ultimate disease expression. Missense mutations associated with the gene encoding the $\alpha$ chain of type XI collagen (COL1A1) and type II collagen (COL2A1) are classic examples of dominant-negative alleles as they disrupt the hetero- or homo-trimer confirmation of collagen subunits and are associated with severe disease phenotypes of osteogenesis imperfecta (OI) type II-IV and spondylepiphyeal dysplasia, respectively. In contrast, all truncating mutations in both genes have been shown to result in haploinsufficiency owing to NMD and are associated with the milder clinical phenotypes of OI type I by $5^{\prime}$ PTC COL $1 A 1^{40,41}$ and Stickler syndrome by $5^{\prime}$ PTC COL $2 A 1 .^{42}$ In both genes, a role for NMD in the ultimate disease phenotype has been postulated; however, experimental studies verifying such a mechanism for these mutant genes (ie that NMD mitigates the dominant-negative effect of the truncated proteins) have not been published.

The extent of the beneficial effect may vary depending on both the toxicity of truncated proteins encoded by different genes and the nature of traits. Rarely, in some instances (Table 3), PTCs triggering NMD may result in more severe diseases by abrogating the hypomorphic function of a mutant protein as illustrated by nonsense mutations in HEXA causing Tay-Sachs disease and the hemizygous X-linked Duchene muscular dystrophy (DMD) gene mutated in muscular dystrophy. Eight frameshift mutations in HEXA resulting in early PTCs susceptible to NMD have been found in $80 \%$ of the carriers of the severe form of Tay-Sachs disease from the Ashkenazi Jewish population. ${ }^{43}$ Whereas the majority of disease-causing mutations in HEXA are associated with the severe form of Tay-Sachs disease, rare missense mutations with some residual activity result in a less severe form of this disorder. Although there has not been any study showing these truncating mutants retain residual activity when NMD is inhibited, the lack of HEXA mRNA owing to NMD can have a detrimental effect in this disorder.

The rare truncating mutations that occur near the $3^{\prime}$ end of dystrophin gene result in variable mild phenotypes, suggesting that these truncating proteins are capable of a partial rescue of the DMD phenotype. ${ }^{44}$ The $5^{\prime}$ PTCs, however, are associated with a severe form of DMD and fail to rescue the phenotype because of the NMD pathway. Evidence that NMD can increase the severity of DMD is a phenotypic rescue of the mouse model of DMD by using the cDNA transgene that encodes a dystrophin with C-terminal truncating mutations. ${ }^{45}$

In theory, the disruption of NMD might also be a logical means for therapy; ${ }^{46}$ for example, in the rare DMD patients with C-terminal truncating mutations with residual activity. It is likely, however, that specific inhibition of NMD might result in undesirable side effects as it may do more harm than the disease by the production of other mutant proteins, such as those caused by possible translational errors of even normal transcripts or translation of trun- cated pseudogene products, resulting in cellular toxicity from synthesis of aberrant proteins. ${ }^{16,47}$ This has created a unique dependence of mammals on the key factors of NMD and its pathway for viability as is implied by the embryonic lethality caused by congenital suppression of NMD factors in mice and the utilization of the NMD pathway in the regulation of normal transcripts. ${ }^{15-18,47,48}$

\section{Conclusion}

In humans, the role of NMD as a modifier of the phenotypic consequences of PTC is becoming more apparent. The identification and characterization of the NMD machinery has provided insight regarding the basic process and development of possible therapeutic reagents by modulating the NMD pathway. Nevertheless, it is important to demonstrate experimentally the role for NMD to enable accurate conclusions regarding phenotypic differences conveyed by truncating mutations. Ultimately, the phenotypic outcome depends on the function of mutant protein and escape from NMD does not necessarily result in a more severe phenotype.

One can predict that NMD may play a role in phenotypic heterogeneity when either distinct phenotypic traits or distinct modes of inheritance (ie dominant versus recessive) are conveyed by allelic truncating mutations. NMD must also be considered when in vitro protein functional assays of disease associated PTC mutation using cDNA truncating clones show a similar outcome for distinct mutations that convey divergent phenotypes. Experimentally, if suppressing important NMD factors, such as hUpf1 and hUpf2, that can inhibit the NMD pathway directly ${ }^{16,20,47}$ results in different expression levels of truncated proteins, then NMD is likely the predominate mechanisms involved. However, each case requires careful experimentation to elucidate the pathogenic mechanisms. It is difficult to estimate the number of genetic diseases in which NMD provides a protective effect (ie partially mitigates the consequences of mutation) owing to phenotypic variability and limited clinical data in the literature for various genetic disorders. However, recent findings not only increase our understanding of the physiological role of NMD but also shed light into the pathogenesis of different aspects of the phenotypes of genetic diseases commonly influenced by NMD.

An important outcome of NMD studies is that many genetic diseases that were previously thought to be the result of dominant-negative mutations on the basis of functional assays may actually result from loss-of-function or haploinsufficiency in vivo because of NMD. ${ }^{20}$ Considering NMD enables a more accurate in vivo genotypephenotype correlation for truncating mutations. However, triggering NMD does not always convert a dominantnegative or gain-of-function mutation into a null allele and 
result in a milder phenotype because some truncated mutant proteins may retain residual wild-type activity. Approaches to protect transcripts containing a premature stop codon from NMD could potentially be used as an alternative treatment for some patients with disease caused by PTC mutations. However, such a therapeutic approach may have other unwanted consequences. NMD likely evolved owing to its beneficial role in eliminating truncated proteins that may have harmful effects on the cells. Nonetheless, NMD must be considered when formulating and testing hypotheses concerning heterogeneous clinical outcomes for different mutations in the same gene. The potential effect of NMD is also important to consider when one encounters a truncating mutation and is attempting to provide the appropriate genetic counseling regarding disease prognosis.

\section{Acknowledgements}

We apologize to colleagues whose work we could not cite owing to limitation of space. We thank Dr Miles Wilkinson for his critical review. This study was supported in part by research grant $(16 B-1)$ for Nervous and Mental Disorders from Ministry of Health, Labor, and Welfare, and a Grant-in-Aid for Scientific Research (17390102) from Japan Society for the promotion of Science, Japan to KI and grants from the United States National Institute for Neurological Disorders and Strokes, NIH (R01 NS27042) (JRL), the Muscular Dystrophy Association and the Charcot-Marie-Tooth Association to JRL.

\section{References}

1 Conti E, Izaurralde E: Nonsense-mediated mRNA decay: molecular insights and mechanistic variations across species. Curr Opin Cell Biol 2005; 17: 316-325.

2 Cui Y, Hagan KW, Zhang S, Peltz SW: Identification and characterization of genes that are required for the accelerated degradation of mRNAs containing a premature translational termination codon. Genes Dev 1995; 9: 423-436.

3 Leeds P, Peltz SW, Jacobson A, Culbertson MR: The product of the yeast UPF1 gene is required for rapid turnover of mRNAs containing a premature translational termination codon. Genes Dev 1991; 5: 2303-2314.

4 Page MF, Carr B, Anders KR, Grimson A, Anderson P: SMG-2 is a phosphorylated protein required for mRNA surveillance in Caenorhabditis elegans and related to Upf1p of yeast. Mol Cell Biol 1999; 19: 5943-5951.

5 Serin G, Gersappe A, Black JD, Aronoff R, Maquat LE: Identification and characterization of human orthologues to Saccharomyces cerevisiae Upf2 protein and Upf3 protein (Caenorhabditis elegans SMG-4). Mol Cell Biol 2001; 21: 209-223.

6 Wagner E, Lykke-Andersen J: mRNA surveillance: the perfect persist. J Cell Sci 2002; 115: 3033-3038.

7 He F, Brown AH, Jacobson A: Upf1p, Nmd2p, and Upf3p are interacting components of the yeast nonsense-mediated mRNA decay pathway. Mol Cell Biol 1997; 17: 1580-1594.

8 Mendell JT, ap Rhys CM, Dietz HC: Separable roles for rent1/ hUpf1 in altered splicing and decay of nonsense transcripts. Science 2002; 298: 419-422.

9 Le Hir H, Izaurralde E, Maquat LE, Moore MJ: The spliceosome deposits multiple proteins 20-24 nucleotides upstream of mRNA exon-exon junctions. EMBO J 2000; 19: 6860-6869.
10 Le Hir H, Izaurralde E, Maquat LE: Pre-mRNA splicing alters mRNP composition: evidence for stable association of proteins at exon-exon junctions. Genes Dev 2000; 14: 1098-1108.

11 Carter MS, Li S, Wilkinson MF: A splicing-dependent regulatory mechanism that detects translation signals. EMBO $J$ 1996; 15: $5965-5975$.

12 Nagy E, Maquat LE: A rule for termination-codon position within intron-containing genes: when nonsense affects RNA abundance. Trends Biochem Sci 1998; 23: 198-199.

13 Ishigaki Y, Li X, Serin G, Maquat LE: Evidence for a pioneer round of mRNA translation: mRNAs subject to nonsense-mediated decay in mammalian cells are bound by CBP80 and CBP20. Cell 2001; 106: 607-617.

14 Lykke-Andersen J, Shu MD, Steitz JA: Human Upf proteins target an mRNA for nonsense-mediated decay when bound downstream of a termination codon. Cell 2000; 103: 1121-1131.

15 Lejeune F, Maquat LE: Mechanistic links between nonsensemediated mRNA decay and pre-mRNA splicing in mammalian cells. Curr Opin Cell Biol 2005; 17: 309-315.

16 Mendell JT, Sharifi NA, Meyers JL, Martinez-Murillo F, Dietz HC: Nonsense surveillance regulates expression of diverse classes of mammalian transcripts and mutes genomic noise. Nat Genet 2004; 36: 1238

17 Kaygun H, Marzluff WF: Regulated degradation of replicationdependent histone mRNAs requires both ATR and Upf1. Nat Struct Mol Biol 2005; 12: 794-800.

18 Kim YK, Furic L, Desgroseillers L, Maquat LE: Mammalian Staufen1 recruits Upf1 to specific mRNA $3^{\prime}$ UTRs so as to elicit mRNA decay. Cell 2005; 120: 195-208.

19 Rajavel KS, Neufeld EF: Nonsense-mediated decay of human HEXA mRNA. Mol Cell Biol 2001; 21: 5512-5519.

20 Inoue $\mathrm{K}$, Khajavi M, Ohyama $\mathrm{T}$ et al: Molecular mechanism for distinct neurological phenotypes conveyed by allelic truncating mutations. Nat Genet 2004; 36: 361-369.

21 Harries LW, Bingham C, Bellanne-Chantelot C, Hattersley AT, Ellard S: The position of premature termination codons in the hepatocyte nuclear factor -1 beta gene determines susceptibility to nonsense-mediated decay. Hum Genet 2005; 118: 214-224.

22 Wang J, Gudikote JP, Olivas OR, Wilkinson MF: Boundaryindependent polar nonsense-mediated decay. EMBO Rep 2002; 3: $274-279$.

23 Frischmeyer PA, Dietz HC: Nonsense-mediated mRNA decay in health and disease. Hum Mol Genet 1999; 8: 1893-1900.

24 Mendell JT, Dietz HC: When the message goes awry: diseaseproducing mutations that influence mRNA content and performance. Cell 2001; 107: 411-414.

25 Forget BG, Benz Jr EJ, Skoultchi A, Baglioni C, Housman D: Absence of messenger RNA for beta globin chain in beta(0) thalassaemia. Nature 1974; 247: 379-381.

26 Baserga SJ, Benz Jr EJ: Nonsense mutations in the human betaglobin gene affect mRNA metabolism. Proc Natl Acad Sci USA 1988; 85: 2056-2060.

27 Pusch M: Myotonia caused by mutations in the muscle chloride channel gene CLCN1. Hum Mutat 2002; 19: 423-434.

28 Rosenfeld PJ, Cowley GS, McGee TL, Sandberg MA, Berson EL, Dryja TP: A null mutation in the rhodopsin gene causes rod photoreceptor dysfunction and autosomal recessive retinitis pigmentosa. Nat Genet 1992; 1: 209-213.

29 Rivolta C, Berson EL, Dryja TP: Dominant Leber congenital amaurosis, cone-rod degeneration, and retinitis pigmentosa caused by mutant versions of the transcription factor CRX. Hum Mutat 2001; 18: 488-498.

30 Patton MA, Afzal AR: Robinow syndrome. J Med Genet 2002; 39: $305-310$.

31 Schwabe GC, Tinschert S, Buschow C et al: Distinct mutations in the receptor tyrosine kinase gene ROR2 cause brachydactyly type B. Am J Hum Genet 2000; 67: 822-831.

32 Gong Y, Chitayat D, Kerr B et al: Brachydactyly type B: clinical description, genetic mapping to chromosome 9q, and 
evidence for a shared ancestral mutation. Am J Hum Genet 1999; 64: 570-577.

33 van Bokhoven $\mathrm{H}$, Celli J, Kayserili $\mathrm{H}$ et al: Mutation of the gene encoding the ROR2 tyrosine kinase causes autosomal recessive Robinow syndrome. Nat Genet 2000; 25: 423-426.

$34 \mathrm{Hu}$ X, Peek R, Plomp A et al: Analysis of the frequent R1141X mutation in the ABCC6 gene in Pseudoxanthoma elasticum. Invest Ophthalmol Vis Sci 2003; 44: 1824-1829.

35 Plomp AS, Hu X, de Jong PT, Bergen AA: Does autosomal dominant Pseudoxanthoma elasticum exist? Am J Med Genet 2004; 126: $403-412$.

36 Wong MH, Filbin MT: Dominant-negative effect on adhesion by myelin Po protein truncated in its cytoplasmic domain. J Cell Biol 1996; 134: 1531-1541.

37 Yoshida M, Colman DR: Rapid functional analysis in Xenopus oocytes of Po protein adhesive interactions. Neurochem Res 2001; 26: $703-712$.

38 Shames I, Fraser A, Colby J, Orfali W, Snipes GJ: Phenotypic differences between peripheral myelin protein-22 (PMP22) and myelin protein zero $(P O)$ mutations associated with CharcotMarie-Tooth-related diseases. J Neuropathol Exp Neurol 2003; 62: $751-764$.

39 Khajavi $\mathrm{M}$, Inoue $\mathrm{K}$, Wiszniewski $\mathrm{W}$, Ohyama $\mathrm{T}$, Snipes GJ, Lupski JR: ER retention and aggregation induced apoptosis associated with neuropathy causing MPZ truncating mutants are abrogated by curcumin treatment. Am J Hum Genet 2005; 77: $841-850$.

40 Körkkö J, Ala-Kokko L, De Paepe A, Nuytinck L, Earley J, Prockop DJ: Analysis of the COL1A1 and COL1A2 genes by PCR amplification and scanning by conformation-sensitive gel electrophoresis identifies only COL1A1 mutations in 15 patients with osteogenesis imperfecta type I: identification of common sequences of null-allele mutations. Am J Hum Genet 1998; 62: 98-110.

41 Willing MC, Deschenes SP, Slayton RL, Roberts EJ: Premature chain termination is a unifying mechanism for COL1A1 null alleles in osteogenesis imperfecta type I cell strains. Am J Hum Genet 1996; 59: 799-809.

42 Snead MP, Yates JR: Clinical and molecular genetics of Stickler syndrome. J Med Genet 1999; 36: 353-359.

43 Myerowitz R: Tay-Sachs disease-causing mutations and neutral polymorphisms in the Hex A gene. Hum Mutat 1997; 9: 195-208.

44 Kerr TP, Sewry CA, Robb SA, Roberts RG: Long mutant dystrophins and variable phenotypes: evasion of nonsensemediated decay? Hum Genet 2001; 109: 402-407.

45 Crawford GE, Faulkner JA, Crosbie RH, Campbell KP, Froehner SC, Chamberlain JS: Assembly of the dystrophin-associated protein complex does not require the dystrophin $\mathrm{COOH}$-terminal domain. J Cell Biol 2000; 150: 1399-1410.
46 Holbrook JA, Neu-Yilik G, Hentze MW, Kulozik AE: Nonsensemediated decay approaches the clinic. Nat Genet 2004; 36: 801-808.

47 He F, Li X, Spatrick P, Casillo R, Dong S, Jacobson A: Genomewide analysis of mRNAs regulated by the nonsense-mediated and $5^{\prime}$ to $3^{\prime}$ mRNA decay pathways in yeast. Mol Cell 2003; 12: $1439-1452$.

48 Medghalchi SM, Frischmeyer PA, Mendell JT, Kelly AG, Lawler AM, Dietz HC: Rent1, a trans-effector of nonsense-mediated mRNA decay, is essential for mammalian embryonic viability. Hum Mol Genet 2001; 10: 99-105.

49 Sung $\mathrm{CH}$, Davenport CM, Nathans J: Rhodopsin mutations responsible for autosomal dominant retinitis pigmentosa. Clustering of functional classes along the polypeptide chain. $J$ Biol Chem 1993; 268: 26645-26649.

50 Tassabehji M, Metcalfe K, Donnai D et al: Elastin: genomic structure and point mutations in patients with supravalvular aortic stenosis. Hum Mol Genet 1997; 6: 1029-1036.

51 Petruzzella V, Panelli D, Torraco A, Stella A, Papa S: Mutations in the NDUFS4 gene of mitochondrial complex I alter stability of the splice variants. FEBS Lett 2005; 579: 3770-3776.

52 Jenkins PB, Abou-Alfa GK, Dhermy D et al: A nonsense mutation in the erythrocyte band 3 gene associated with decreased mRNA accumulation in a kindred with dominant hereditary spherocytosis. J Clin Invest 1996; 97: 373-380.

53 Toye AM, Bruce LJ, Unwin RJ, Wrong O, Tanner MJ: Band 3 Walton, a C-terminal deletion associated with distal renal tubular acidosis, is expressed in the red cell membrane but retained internally in kidney cells. Blood 2002; 99: 342-347.

54 Yang Y, Hentati A, Deng HX et al: The gene encoding alsin, a protein with three guanine-nucleotide exchange factor domains, is mutated in a form of recessive amyotrophic lateral sclerosis. Nat Genet 2001; 29: 160-165.

55 Gros-Louis F, Meijer IA, Hand CK et al: An ALS2 gene mutation causes hereditary spastic paraplegia in a Pakistani kindred. Ann Neurol 2003; 53: 144-145.

56 Johnston JJ, Olivos-Glander I, Killoran C et al: Molecular and clinical analyses of Greig cephalopolysyndactyly and PallisterHall syndromes: robust phenotype prediction from the type and position of GLI3 mutations. Am J Hum Genet 2005; 76: 609-622.

57 Tzoulaki I, White IM, Hanson IM: PAX6 mutations: genotypephenotype correlations. BMC Genet 2005; 6: 27.

58 Alonso J, Garcia-Miguel P, Abelairas J et al: Spectrum of germline RB1 gene mutations in Spanish retinoblastoma patients: phenotypic and molecular epidemiological implications. Hum Mutat 2001; 17: 412-422.

59 Li A, Swift M: Mutations at the ataxia-telangiectasia locus and clinical phenotypes of A-T patients. Am J Med Genet 2000; 92: $170-177$. 